\title{
Reconocimiento de Acciones Humanas con Mínima Información Disponible mediante Transferencia de Aprendizaje y Modelos Ocultos de Markov
}

\author{
M. Rodríguez, C. Orrite \\ Laboratorio de Visión por Computador (CVlab) \\ Instituto de Investigación en Ingeniería de Aragón (I3A) \\ Universidad de Zaragoza, Mariano Esquillor s/n, 50018, Zaragoza, Spain. \\ Tel. +34-976762707, Fax +34-976762043, e-mail:mrodrigo@unizar.es
}

\begin{abstract}
La puesta en funcionamiento de un sistema de reconocimiento de acciones humanas requiere de un periodo de entrenamiento para que el sistema aprenda las acciones deseadas. Como cada escenario de desarrollo tiene unas características específicas, los sistemas actuales suelen realizar dicho entrenamiento para cada escenario. Esto supone que la puesta en funcionamiento de un sistema puede demorarse en el tiempo debido principalmente a dos factores: (i) la necesidad de tener suficiente información disponible en cada nuevo escenario, para ello es necesario grabar repeticiones de las diferentes acciones deseadas una vez instalados los dispositivos de grabación, (ii) el tiempo de procesamiento que supone un entrenamiento nuevo, aunque con la continua mejora de los procesadores puede no ser un factor excesivamente restrictivo. Lo que nosotros planteamos es una nueva metodología que reduzca el tiempo de entrenamiento debido al primer factor sin rebajar las prestaciones. Para ello proponemos el diseño de un sistema que desde la primera grabación disponible de una acción es capaz de entrenar un reconocedor con prestaciones aceptables mediante el uso de información extraida de escenarios anteriormente conocidos y aplicada a una modificación de un sistema de reconocimiento basado en Modelos Ocultos de Markov (HMM). Esto permite realizar un entrenamiento previo reduciendo el procesamiento y las necesidades de información en el nuevo escenario. Nuestro sistema realiza un reconocimiento basado en una variación de HMM discretos, llamada Fuzzy Observation Hidden Markov Model (FO-HMM), utilizando un diccionario de características espacio-temporales creado mediante un proceso de transferencia de aprendizaje desde bases de datos conocidas.
\end{abstract}

